\title{
Exchange-biased AMR bridges for magnetic field sensing and biosensing
}

\author{
Hansen, Mikkel Fougt; Rizzi, Giovanni
}

Published in:

I E E Transactions on Magnetics

Link to article, DOI:

10.1109/TMAG.2016.2614012

Publication date:

2017

Document Version

Peer reviewed version

Link back to DTU Orbit

Citation (APA):

Hansen, M. F., \& Rizzi, G. (2017). Exchange-biased AMR bridges for magnetic field sensing and biosensing. I $E$ E E Transactions on Magnetics, 53(4). https://doi.org/10.1109/TMAG.2016.2614012

\section{General rights}

Copyright and moral rights for the publications made accessible in the public portal are retained by the authors and/or other copyright owners and it is a condition of accessing publications that users recognise and abide by the legal requirements associated with these rights.

- Users may download and print one copy of any publication from the public portal for the purpose of private study or research.

- You may not further distribute the material or use it for any profit-making activity or commercial gain

- You may freely distribute the URL identifying the publication in the public portal

If you believe that this document breaches copyright please contact us providing details, and we will remove access to the work immediately and investigate your claim 


\title{
Exchange-biased AMR bridges for magnetic field sensing and biosensing
}

\author{
Mikkel Fougt Hansen ${ }^{1}$ and Giovanni Rizzi ${ }^{1}$ \\ ${ }^{1}$ Department of Micro- and Nanotechnology, DTU Nanotech, Building 345B, \\ Technical University of Denmark, DK-2800 Kongens Lyngby, Denmark
}

\begin{abstract}
We introduce magnetic field sensor bridges that are formed by combinations of stripes of an exchange-pinned magnetic stack displaying anisotropic magnetoresistance. We present a systematic overview on how the stripe geometries can be combined to form sensor bridges with a scalable signal and how these can be tailored towards detection of external magnetic fields and of magnetic beads over or tethered to the sensor surface. Particular attention is given to the case where the beads are magnetized by the sensor self-field due to the bias current passed through the sensor, which is interesting for magnetic bead sensing and where the static and dynamic magnetic bead response can be monitored in the 2 nd harmonic sensor response to an oscillating bias current. The recent literature on applications of these sensors for detection of magnetic fields and of the dynamic and static response of magnetic beads in suspension and attached to the sensor surface is reviewed as well as the use of the sensors for magnetic biosensing in volumeand surface-based formats. We illustrate that the sensors can be flexibly designed and applied for a number of sensing applications with sensitive detection of magnetic fields down to the nT range.
\end{abstract}

Index Terms-Magnetoresistive sensor, planar Hall effect, magnetic field sensor, magnetic biosensor.

$\mathbf{M}$ AGNETIC field sensors based on the anisotropic magnetoresistance (AMR) effect have been used for magnetic field sensing since the 90s [1]-[4] and have in the last decade attracted renewed interest. Compared to the predominant giant magnetoresistance sensors they offer a lower signal level but they provide advantages of simpler fabrication, flexibility in choice of device shape and resistance and a high signal-to-noise ratio at low frequencies [5]-[7]. Therefore, they are still attractive for several applications and their use for low-field sensing in, e.g., compasses in mobile phones and satellites, is widespread [8].

AMR sensors can be divided into two main classes. In the first class, the sensors are in the form of crosses that share geometry with Hall sensors. In these a current is injected along one direction in the sensor cross and the voltage difference is measured in the orthogonal direction. The voltage output from a sensor cross is given by the off-diagonal elements in the resistivity tensor and they were therefore been termed planar Hall effect (PHE) sensors. PHE sensors generally produce a low signal but have a high signal-to-noise ratio and they can be optimized to detect sub-nT magnetic fields at low frequencies [6], [7]. To realize the full potential of PHE sensors, however, ultra-low-noise readout electronics is required. In the second class, four AMR elements are combined to form a Wheatstone bridge where the current is still injected along one direction and the voltage is measured in the orthogonal direction [1], [2]. Several geometries of these are available [1], where the most widespread commercial sensors are based on the socalled barber pole design [4].

Exchange-biased PHE sensor crosses were introduced for detection of magnetic beads in 2004 by Ejsing et al. [9] and spawned a renewed interest in AMR sensors, now with a

(email: focus on magnetic bead detection and magnetic biosensing [9], [10]. To avoid the need for special low-noise amplification electronics and to increase the sensor signal, Henriksen et al. [11] and Oh et al. [12] introduced Wheatstone bridge geometries of the same exchange-biased stack as used for the PHE sensors. These produced the same dependence of the signal on the magnetization orientation as the PHE sensors, i.e., the signal was given by the off-diagonal elements of the resistivity tensor, but a substantial geometrical signal amplification was obtained. To emphasize the strong kindship between PHE sensors and Wheatstone bridge sensors made from the same exchange-biased stack, Henriksen et al. [11] introduced the term 'planar Hall effect bridge' (PHEB) sensors to distinguish these from other AMR bridge sensors, such as barber pole sensors. This term, although the sensors may more correctly be referred to as 'exchange-biased AMR bridge sensors', was used in subsequent work and will also to some extent be used below. The bridge design has enabled users to better exploit the intrinsically high signal-to-noise ratio for AMR sensors and it has further significantly expanded the sensor design space such that the exchange-biased AMR bridge/PHEB design can be tailored to provide optimal signal for given applications.

Here, we present an overview of the construction and reported applications of exchange-biased AMR bridge/PHEB sensors tailored for magnetic field sensing with a special focus on magnetic bead sensing in lab-on-a-chip systems for dynamic magnetic susceptibility measurements and for biosensing applications.

\section{THEORY}

In this section, we first describe the structure of the magnetic stacks used for the sensors. Then, we use energy minimization to obtain a single domain description for the magnetic field response of a stripe (Fig. 1a). We introduce the contributions 
to the magnetic field experienced by a stripe, especially in presence of magnetic beads. We will put particular emphasis on the detection of magnetic beads magnetized by the sensor self-field arising from the bias current passed through the sensor. Finally, we illustrate how stripes can be combined to form sensor bridges with different properties and we present expressions for the bridge signals obtained using lock-in detection. In this manuscript, we will generally write magnetic fields in terms of $\mathbf{B}$-fields $\left(=\mu_{0} \mathbf{H}\right)$ to simplify notation.

\section{A. Sensor stack}

The sensors are generally made of a stack with magnetically active layers (bottom-to-top) $\mathrm{Ni}_{80} \mathrm{Fe}_{20}\left(t_{\mathrm{fm}}\right) / \mathrm{Cu}\left(t_{\mathrm{Cu}}\right) /$ $\mathrm{Mn}_{80} \mathrm{Ir}_{20}\left(t_{\mathrm{afm}}\right)$ deposited on an oxidized Silicon substrate. Additional layers include a Ta buffer layer (typical thickness 3-15 nm) and a Ta capping layer (typical thickness 3-5 nm). Here, the ferromagnetic (fm) Ni-Fe layer is the active magnetoresistive layer and the antiferromagnetic (afm) Mn-Ir layer is used to pin the magnetization of the fm layer along the $x$ direction in Fig. 1a to achieve a unique single domain magnetic state in zero external magnetic field. This pinning direction is defined by applying a magnetic field during the thin film deposition. Typical values are $t_{\mathrm{fm}}=10-30 \mathrm{~nm}, t_{\mathrm{afm}}=10-20$ and $t_{\mathrm{Cu}}=0-1.2 \mathrm{~nm}$, see Table III. The $\mathrm{Cu}$ layer has been introduced to weaken the exchange-pinning of the fm layer to increase the response to an external magnetic field [10], [13], [14] and is only used in later studies. In addition to the above bi- or tri-layer stacks, a spin-valve stack has also been used in a few studies, where a Ni-Fe layer is added between the $\mathrm{Cu}$ and Mn-Ir layers [10], [12].

\section{B. Single domain model for magnetic response}

The angle $\theta$ of the magnetization for a single domain stripe (Fig. 1a) with magnetization $M_{\mathrm{s}}$ can be found by minimizing the magnetic energy. In a magnetic field $B_{y}$ acting along the $y$-direction, the magnetic energy density $u$ for a branch of angle $\alpha$ can be written as

$u / M_{\mathrm{s}}=-B_{y} \sin \theta-B_{\mathrm{ex}} \cos \theta-\frac{1}{2} B_{\mathrm{K}} \cos ^{2} \theta-\frac{1}{2} B_{\mathrm{sh}} \cos ^{2}(\alpha-\theta)$,

where $B_{\text {ex }}$ is the exchange-pinning field, $B_{\mathrm{K}}$ is the anisotropy field, and $B_{\text {sh }}$ is the shape anisotropy field [15]. The exchange and anisotropy energy contributions are minimal when $\mathbf{M}$ is along the $x$-axis $(\theta=0)$, whereas the shape anisotropy contribution is minimal when $\mathbf{M}$ is along the length of the stripe $(\theta=\alpha$ or $\alpha+\pi)$. Thus, it is clear that when the shape anisotropy is not negligible, the value of $\theta$ should be found separately for all possible stripe orientations. For negligible shape anisotropy, Taylor expansion of the derivative of the energy density to first order gives that $u$ is minimized for

$$
\theta \approx B_{y} /\left(B_{\mathrm{ex}}+B_{\mathrm{K}}\right) .
$$

Thus, in this case the magnetization rotation $\theta$ is proportional to $B_{y}$ for low magnetic fields.
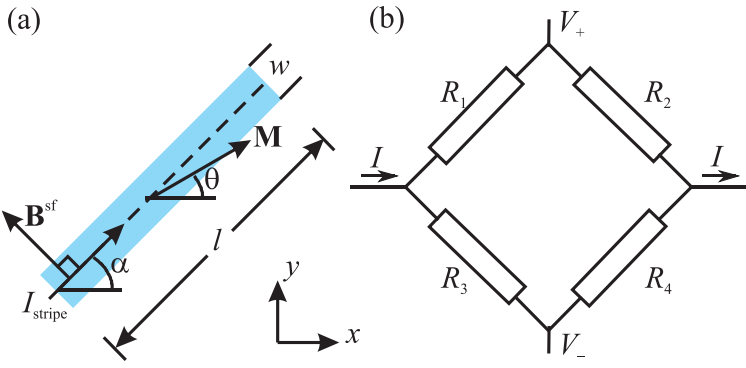

Fig. 1. (a) Illustration of sensor stripe with definitions of geometrical parameters and coordinate system. (b) Wheatstone bridge readout configuration with indication of the positive and negative voltage terminals for the bridge voltage $V$.

\section{Contributions to magnetic field}

We consider the average total magnetic field $B_{y}$ acting on a sensor stripe in the $y$-direction. The contributions to $B_{y}$ can be divided into a contribution $B_{y}^{\text {ext }}$ due to a homogeneous external magnetic field $B_{y}^{\text {app }}$ applied along the $y$-direction and a contribution from the magnetic field induced by the bias current passing through the sensor (the self-field), $B_{y}^{\text {sf }}$, i.e.,

$$
B_{y}=B_{y}^{\mathrm{ext}}+B_{y}^{\mathrm{sf}} .
$$

When magnetic beads are present over the sensor, they are magnetized by $B_{y}^{\text {app }}$ and we can write

$$
B_{y}^{\text {ext }}=B_{y}^{\text {app }}(1+\beta \chi)
$$

where $\beta$ is a factor accounting for the perturbation of the external magnetic field experienced by the sensor due to the presence of magnetic beads and $\chi$ is the complex magnetic susceptibility of the beads. $\beta$ depends on both the amount and distribution of the magnetic beads as well as on the geometry of the sensor. When the beads are magnetized by an external magnetic field, their magnetic dipoles are all aligned along the field. The magnetic field directly under a magnetic bead has a direction opposite to the dipole orientation whereas the magnetic field in front of the dipole is directed along the dipole. This causes both the sign and magnitude of the signal to depend on the detailed arrangement of the beads over the sensor as well as on the height profile of the sensor [16] and in the extreme case of a uniform bead distribution in the halfspace over the sensor, zero signal is expected [16], [17]. These magnetic bead signal cancellation effects can be mitigated by careful sensor design or by selective functionalization of either the sensor area or the area outside the sensor [16]. In our work, we have not pursued measurements of $\beta$, but we maintain $\beta$ in the description below to keep the description general and consistent.

In our application of PHEBs, we magnetize the magnetic beads using the self-field arising from the applied bias current. The self-field circulates around the sensor stripe and thus the magnetic dipole of a magnetic bead changes orientation when it is moved from being over the sensor stripe to outside the sensor stripe. Fig. 2a shows the magnitude of the magnetic field over a sensor stripe at a typical experimental condition. For a stripe with $\alpha=0$, the magnetizing self-field is oriented in the negative $y$-direction and a magnetic bead placed over the 

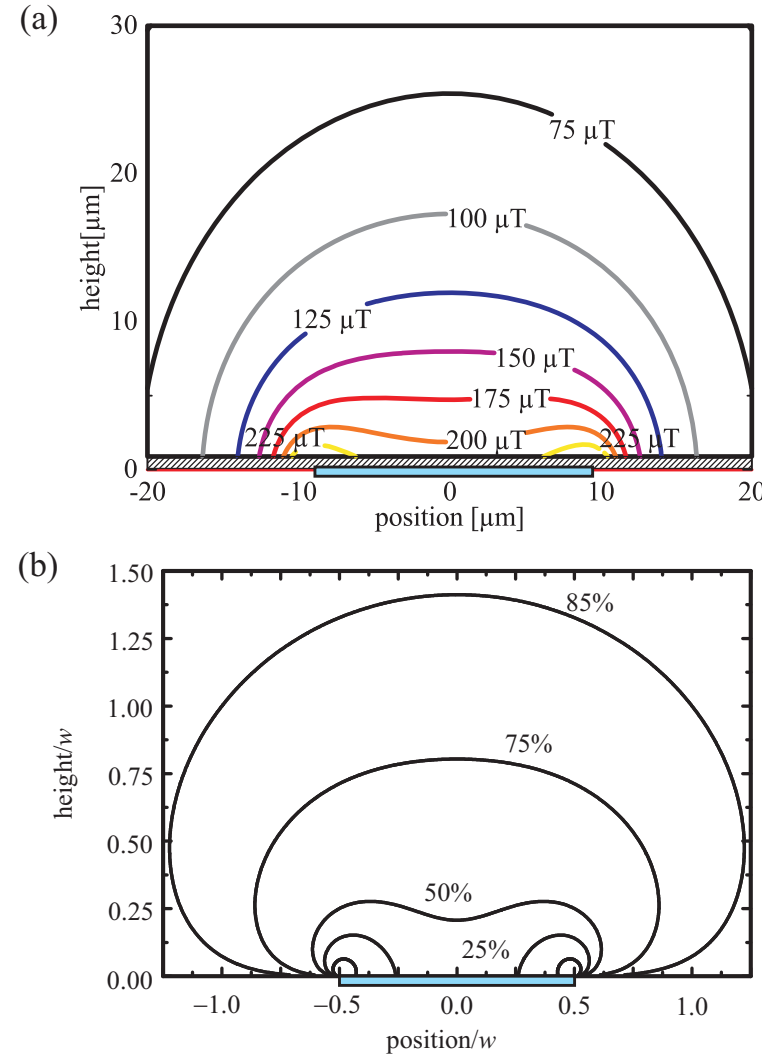

Fig. 2. (a) Magnitude of self-field over a stripe of width $w=20 \mu \mathrm{m}$ carrying a current of $7 \mathrm{~mA}$. Adapted from [18] with permission of AIP publishing. (b) Cummulative signal from homogeneous distribution of magnetic beads in half-space over stripe. Adapted from [19] with permission of AIP publishing. In both graphs, the position of the sensor stripe is indicated by the blue bar.

stripe thus gives rise to a magnetic field acting on the stripe in the positive $y$-direction. When a magnetic bead is positioned away from the center of the stripe, its dipole moment is aligned with the magnetizing field, with a $z$-axis component. A detailed analysis of the magnetic field as function of the bead position has revealed that, in this case, the magnetic beads contribute to the signal with the same sign irrespective of their position [19]. Thus, the signal from magnetic beads magnetized by the sensor self-field does not suffer from the cancellation effect that appears for magnetic beads magnetized by a homogeneous magnetic field. The contribution due to the self-field along the $y$-direction can be written as

$$
B_{y}^{\text {sf }}=I_{\text {stripe }}\left(\gamma_{0}+\gamma_{1} \chi\right) \cos \alpha=B^{\text {sf }} \cos \alpha,
$$

where $I_{\text {stripe }}$ is the current passed through the stripe, $\gamma_{0}$ is a constant accounting for self-bias due to current shunting in other layers than that exhibiting AMR and $\gamma_{1}$ is a constant accounting for the volume and distribution of magnetic beads over the sensor [19]. Fig. 2b shows a contour plot of the contribution to the signal from a homogeneous distribution of magnetic beads in a half-space above the sensor stripe. It is observed that about $80-90 \%$ of the signal is due to beads within a radius of about $1.3 \mathrm{w}$ from the sensor center. The corresponding area of the stripe cross-section is about $2.7 w^{2}$ [19]. This means, that most of the signal for a single stripe of length $l=280 \mu \mathrm{m}$ and $w=20 \mu \mathrm{m}$ arises from beads in a volume of $2.7 l w^{2} \approx 0.3 \mathrm{~nL}$.

177

178

\section{Resistance of sensor construction element}

In this section, we present the resistance of the sensor construction element shown in Fig. 1a. The stripe of the magnetic stack has a width $w$, length $l$ and thickness $t$. The angle of a positive current passed through the stripe to the $x$-axis is denoted $\alpha$ and the stripe is assumed to have a homogeneous magnetization oriented at an angle $\theta$ to the $x$ axis. Due to anisotropic magnetoresistance, the resistivity of the stripe depends on the relative orientation of the current and the magnetization. The resistivities when these are parallel and perpendicular are denoted $\rho_{\|}$and $\rho_{\perp}$, respectively, and we define $\Delta \rho \equiv \rho_{\|}-\rho_{\perp}$. Typically, $\Delta \rho$ is about $2-3 \%$ of the average resistivity. Using Ohm's law, it can be shown that the resistance of the single stripe of the magnetic stack shown in Fig. 1a is

$$
R(\alpha, \theta)=R_{0}+\frac{l \Delta \rho}{2 w t} \sin (2 \theta) \sin (2 \alpha),
$$

where $R_{0}=l\left(\rho_{\|}+\rho_{\perp}\right) /(2 w t)$ is the stripe resistance when $\theta=0$ [11], [20], [21].

Inserting the low-field result for $\theta$ from Eq. (2) in Eq. (6) yields

$$
R(\alpha)=R_{0}-\sin (2 \alpha) S_{0} B_{y}
$$

with the single stripe low-field sensitivity

$$
S_{0} \equiv-\frac{l \Delta \rho}{w t\left(B_{\mathrm{ex}}+B_{\mathrm{K}}\right)} .
$$

Further inserting the expressions for the total magnetic field $B_{y}$ from Eqs. (4)-(5) in Eq. (7) gives

$$
R(\alpha)=R_{0}-\sin (2 \alpha)\left(S_{0} B_{y}^{\text {ext }}+S_{0} B^{\text {sf }} \cos \alpha\right) .
$$

From Eq. (9), it is clear that interesting values of $\alpha$ are those where $\sin (2 \alpha)= \pm 1$ or $\cos \alpha= \pm 1$, i.e., $\alpha=p \pi / 4$ with $p$ being an integer number. It is also clear that extrema for $\sin (2 \alpha) \cos \alpha$ are of interest as they correspond to $\alpha$-values that maximize the self-field signal. These are obtained for $\alpha=\arccos ( \pm \sqrt{2 / 3})$ (maxima) and $\alpha=-\arccos ( \pm \sqrt{2 / 3})$ (minima) where $\sin (2 \alpha) \cos \alpha= \pm 0.7698$. Comparing the result for these value to that for $\alpha=\pi / 4$, where $\sin (2 \alpha) \cos \alpha=$ $1 / \sqrt{2} \approx 0.7071$, the improvement is only about $9 \%$. For simplicity we therefore restrict our considerations below to $\alpha=p \pi / 4$. Table I gives an overview of the signs of the contributions to $R$ due to the external field and the self-field for these values.

\section{E. Sensor bridge designs}

The presented elements can be combined to form the four arms $R_{1}-R_{4}$ of a Wheatstone bridge as indicated in Fig. 1b, where the resistance of each arm is given by addition of stripes, $R(\alpha)$, remembering that $\alpha$ should be chosen to represent the direction of a positive applied current through the resistor. The output voltage (potential increase in the $y$-direction) from the bridge is

$$
V=I \frac{R_{2} R_{3}-R_{1} R_{4}}{R_{1}+R_{2}+R_{3}+R_{4}} \approx \frac{1}{2} I\left(R_{3}-R_{1}\right),
$$


TABLE I

OVERVIEW OF THE SIGNS OF THE CONTRIBUTIONS TO THE RESISTANCE $R(\alpha)=R_{0}+k^{\text {ext }} S_{0} B_{y}^{\text {ext }}+k^{\text {sf }} \frac{1}{\sqrt{2}} S_{0} B^{\text {sf }}$ OF A STRIPE DUE TO THE EXTERNAL FIELD AND THE SELF-FIELD AS FUNCTION OF $\alpha$, WHERE $p$ DENOTES AN INTEGER.

\begin{tabular}{c|cc}
$\alpha$ & $k^{\mathrm{ext}}$ & $k^{\mathrm{sf}}$ \\
\hline$-3 \pi / 4+2 p \pi$ & -1 & +1 \\
$-\pi / 4+2 p \pi$ & +1 & +1 \\
$p \pi / 2$ & 0 & 0 \\
$\pi / 4+2 p \pi$ & -1 & -1 \\
$3 \pi / 4+2 p \pi$ & +1 & -1 \\
\hline
\end{tabular}
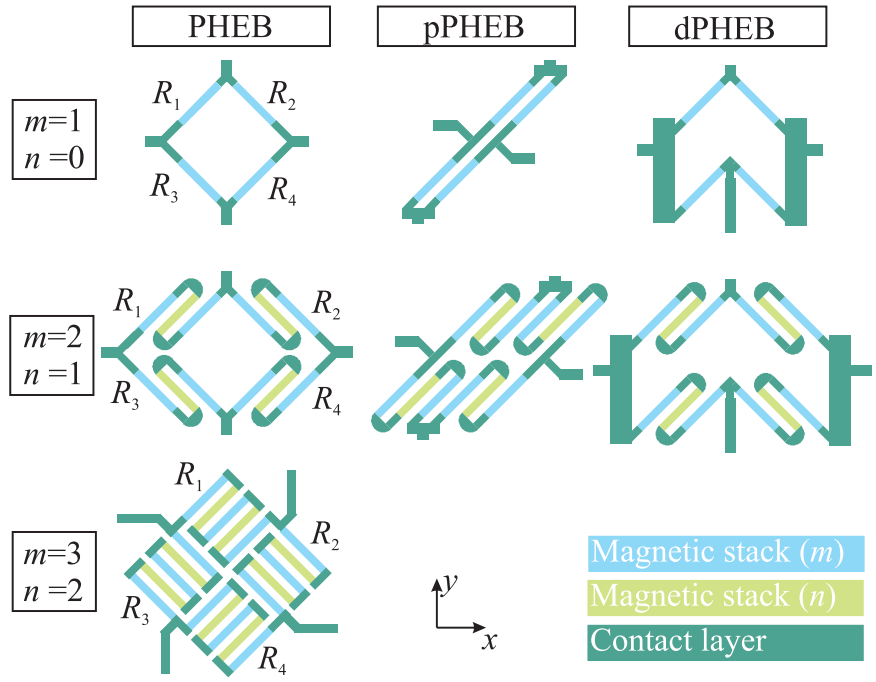

Fig. 3. Illustration of PHEB, pPHEB and dPHEB sensor designs for the indicated values of $m$ (blue stripes with current along $\pi / 4$ in $R_{1}$ and $R_{4}$ ) and $n$ (green stripes with current along $-3 \pi / 4$ in $R_{1}$ and $R_{4}$ ). In the bottom row is illustrated a compact design of the PHEB sensor with $m=3$ and $n=2$.

where the latter result is valid when $R_{1}+R_{2} \approx R_{3}+R_{4}$ where $I_{\text {stripe }}=I / 2$.

By combining in series multiple resistive elements in a meandering fashion, it is possible to increase the sensor length with a minor increase of the sensor footprint. The space of design parameters is large and we therefore only consider a few combinations of those. Below, we follow and generalize the analysis presented in [21]. The low-field bridge output can easily be calculated for all possible combinations and experimental conditions by use of Eq. (10) with $R(\alpha)$ given by Eq. (9) or Table I.

Figure 3 shows the bridge designs that we have employed in our studies. It presents bridges whose arms are a single resistive element or a meander of parallel resistive elements. Let us first consider meandering sensor designs with $R_{1}=R_{4}=m R(\pi / 4)+n R(-3 \pi / 4)$, where $m$ and $n$ are non-negative integers, and let us assume that all resistances experience identical values of $\beta, \gamma_{0}$ and $\gamma_{1}$ corresponding to that they all have the same width, are functionalized identically and are exposed to the same sample. Further, we note that often a meander geometry is designed such that $n=m-1$ or $n=0$.

In the first design, termed PHEB or meander PHEB (mPHEB) (Fig. 3), each element in $R_{1}$ and $R_{4}$ with orientation $\alpha$ is matched by a corresponding element in $R_{2}$ and $R_{3}$ with orientation $-\alpha$, i.e., we let $R_{2}=R_{3}=m R(-\pi / 4)+$ $n R(3 \pi / 4)$ [11], [21]. This design has a high degree of symmetry. The bridge output voltage is

$$
V^{\mathrm{PHEB}}=I(m+n) S_{0} B_{y}^{\mathrm{ext}}+\frac{1}{\sqrt{2}} I(m-n) S_{0} B_{y}^{\mathrm{sf}} .
$$

For the external field contribution, the contributions from the two current orientations in each resistor are additive and the signal is proportional to the total length of the meander $l(m+n)$ in each resistor with an observed low-field sensitivity $S_{0}^{\text {obs }}=(m+n) S_{0}$. For the self-field contribution, however, the contributions from the two current orientations are subtractive and the signal is proportional to $m-n$. Thus, a meander structure does not increase this signal unless one of the current orientations is eliminated $(n=0)$, e.g., by using a nonmagnetoresistive conductor [21].

In the second design, termed parallel PHEB (pPHEB) (Fig. 3 ), each element in $R_{1}$ and $R_{4}$ with orientation $\alpha$ is matched by a corresponding element in $R_{2}$ and $R_{3}$ with orientation $\alpha-\pi$, i.e., we let $R_{2}=R_{3}=m R(-3 \pi / 4)+n R(\pi / 4)$ [21]. Inspecting Table I, this design choice conserves the sign of $k^{\text {ext }}$ but changes the sign of $k^{\text {sf }}$, i.e., it eliminates the contribution from a homogeneous external field in the bridge voltage. It can be thought of as an antisymmetric design. The bridge output voltage is

$$
V^{\mathrm{pPHEB}}=\frac{1}{\sqrt{2}} I(m-n) S_{0} B_{y}^{\mathrm{sf}} .
$$

A third design can be made, which is nominally insensitive to the self-field contribution to the signal such that only the signal due to the external field is detected. Requiring parallel stripes, balanced contributions from the stripes and $k^{\text {ext }}=-1$ for $R_{1}$ and $R_{4}$ and $k^{\text {ext }}=+1$ for $R_{2}$ and $R_{3}$, it observed from Table I that this can be obtained for $R_{1}=R_{4}=m R(-3 \pi / 4)+n R(\pi / 4)$ and $R_{1}=R_{4}=$ $m R(\pi / 4)+n R(-3 \pi / 4)$. Noting that switching the roles of the current and voltage leads corresponds to the transformation $\alpha \rightarrow-\alpha$ and $m \rightarrow n$, we observe that this design can be realized by switching the current and voltage leads of the already presented PHEB design.

A final important design, termed differential PHEB (dPHEB) (Fig. 3), relies on differential detection between the top $\left(R_{1}\right.$ and $\left.R_{2}\right)$ and bottom $\left(R_{3}\right.$ and $\left.R_{4}\right)$ halves of the sensor bridge, where $R_{1}=R_{3}=m R(\pi / 4)+n R(-3 \pi / 4)$ and $R_{2}=R_{4}=m R(-\pi / 4)+n R(3 \pi / 4)$ [20]. Under nominally identical physical conditions (temperature and external magnetic field) and homogeneous amounts of beads on the top and bottom parts, respectively, the bridge voltage from this design is

$V^{\mathrm{dPHEB}}=\frac{1}{2} I(m+n) S_{0} B_{y}^{\mathrm{app}} \Delta \beta \chi+\frac{1}{4 \sqrt{2}} I^{2}(m-n) S_{0} \Delta \gamma_{1} \chi$

with $\Delta \beta \equiv \beta^{\text {top }}-\beta^{\text {bottom }}, \Delta \gamma_{1} \equiv \gamma_{1}^{\text {top }}-\gamma_{1}^{\text {bottom }}$ and where we have used $I_{\text {stripe }} \approx I / 2$. This design can be scaled to more branches, if needed. It produces a signal only due to the magnetic beads and is suited for distinguishing small magnetic bead signals in a background. 
TABLE II

$1^{\text {st }}$ AND $2^{\text {nd }}$ HARMONIC IN-PHASE AND OUT-OF-PHASE SIGNALS CALCULATED FOR THE PRESENTED SENSOR DESIGNS. THE SIGNAL IS OBTAINED BY MULTIPLYING THE LEFT COLUMN WITH THE COLUMN FOR THE RELEVANT SENSOR DESIGN. ADAPTED FROM [21] WITH PERMISSION OF AIP PUBLISHING.

\begin{tabular}{lccc}
\hline Lock-in signal & PHEB & pPHEB & dPHEB \\
\hline$V_{1}^{\prime}=(m+n) S_{0} I_{\mathrm{rms}}$ & $B_{y}^{\text {app }}\left(1+\beta \chi_{0}\right)$ & 0 & $\frac{1}{2} \Delta \beta B_{y}^{\text {app }} \chi_{0}$ \\
$V_{1}^{\prime \prime}=(m+n) S_{0} I_{\mathrm{rms}}$ & 0 & 0 & 0 \\
$V_{2}^{\prime}=-\frac{m-n}{4} S_{0} I_{\mathrm{rms}}^{2}$ & $\gamma_{1} \chi^{\prime \prime}$ & $\gamma_{1} \chi^{\prime \prime}$ & $\frac{1}{2} \Delta \gamma_{1} \chi^{\prime \prime}$ \\
$V_{2}^{\prime \prime}=-\frac{m^{\prime \prime}}{4} S_{0} I_{\mathrm{rms}}^{2}$ & $\gamma_{0}+\gamma_{1} \chi^{\prime}$ & $\gamma_{0}+\gamma_{1} \chi^{\prime}$ & $\frac{1}{2} \Delta \gamma_{1} \chi^{\prime}$ \\
\hline
\end{tabular}

\section{F. Electrical readout and magnetic bead response}

In our work, we have almost exclusively used lock-in detection to read out the sensor response. To do this, an alternating current $I(t)=\sqrt{2} I_{\text {rms }} \cos (\omega t)$ is applied and either the $1^{\text {st }}$ or $2^{\text {nd }}$ harmonic lock-in signal is detected. To simplify the description, we only consider dc external magnetic fields below. The magnetic moment response of a magnetic bead to a magnetic field $H(t)=H_{\mathrm{dc}}+H_{\mathrm{ac}} \cos (\omega t)$ is

$$
m(t)=V_{\text {bead }}\left[H_{\mathrm{dc}} \chi_{0}+H_{\mathrm{ac}}|\chi| \cos (\omega t-\phi)\right],
$$

where $\chi_{0}$ is the dc magnetic susceptibility, $|\chi|$ is the magnitude of the frequency-dependent complex magnetic susceptibility and $\phi$ is the phase lag of the response with respect to the excitation. Using the cosine relations, this can also be written in terms of the in-phase and out-of-phase components of the magnetic susceptibility $\chi=\chi^{\prime}-\mathrm{i} \chi^{\prime \prime}=|\chi|(\cos \phi-\mathrm{i} \sin \phi)$ as

$$
m(t)=V_{\text {bead }}\left[H_{\mathrm{dc}} \chi_{0}+H_{\mathrm{ac}}\left(\chi^{\prime} \cos (\omega t)+\chi^{\prime \prime} \sin (\omega t)\right)\right] .
$$

To include the complex susceptibility in the description of the self-field contribution to the signal, we can therefore make the substitution $\gamma_{1} I_{\mathrm{rms}} \cos (\omega t) \chi \rightarrow \gamma_{1} I_{\mathrm{rms}}\left[\chi^{\prime} \cos (\omega t)+\right.$ $\left.\chi^{\prime \prime} \sin (\omega t)\right]$ in the expressions for the bridge voltage.

The in-phase and out-of-phase components of the $n^{\text {th }}$ harmonic signal from the lock-in amplifier can be calculated as

$$
\begin{aligned}
V_{n}^{\prime} & =\frac{\sqrt{2}}{2 \pi} \int_{-\pi}^{\pi} \cos (n \omega t) V(\omega t) \mathrm{d}(\omega t) \\
V_{n}^{\prime \prime} & =\frac{\sqrt{2}}{2 \pi} \int_{-\pi}^{\pi} \sin (n \omega t) V(\omega t) \mathrm{d}(\omega t) .
\end{aligned}
$$

The values of $V_{n}^{\prime}$ and $V_{n}^{\prime \prime}$ correspond to the coefficients in a Fourier series representation of $V(t)$ divided by $\sqrt{2}$. The results for the presented mPHEB, pPHEB and dPHEB sensors are given in Table II.

Note that with the above definition of the bridge voltage $V$ as the potential increase in the $y$-direction, $V_{1}^{\prime}$ has a negative slope vs. applied field as $S_{0}$ is defined to be negative in Eq. (8). In studies of the field sensitivity, it has often been convenient to define $V$ as the potential drop in the $y$-direction $(=-V)$ to obtain a positive slope of the signal vs. field response. In studies focusing on magnetic bead detection, the definition of $V$ as the potential increase in the $y$-direction has typically been used as the introduction of magnetic nanobeads in this case causes a positive change in $V_{2}$ (cf. Table II with $S_{0}<0$ ).
In this work, we will consider magnetic beads with a remanent magnetic moment that may relax in a liquid via a physical rotation (Brownian relaxation). This magnetic relaxation process can generally be described by the Debye model [22]

$$
\chi=\chi^{\prime}-i \chi^{\prime \prime}=\frac{\chi_{0}-\chi_{\infty}}{1+i f / f_{\mathrm{B}}}+\chi_{\infty},
$$

where $\chi_{\infty}$ is the susceptibility at infinite frequency and $f_{\mathrm{B}}$ is the Brownian relaxation frequency given by

$$
f_{\mathrm{B}}=\frac{k_{\mathrm{B}} T}{\pi^{2} \eta d_{h}^{3}} .
$$

Here, $k_{\mathrm{B}} T$ is the thermal energy, $\eta$ is the viscosity of the liquid in which the beads are suspended and $d_{h}$ is the hydrodynamic diameter of the beads. A distribution of relaxation times can be accounted for using the empirical Cole-Cole model [23] or by integrating over the size distribution [18]. A measurement of the magnetic susceptibility vs. frequency will show $\chi^{\prime} \approx \chi_{0}$ and $\chi^{\prime \prime} \approx 0$ at $f \ll f_{\mathrm{B}}$ and $\chi^{\prime} \approx \chi_{\infty} \ll \chi_{0}$ and $\chi^{\prime \prime} \approx 0$ at $f \gg f_{\mathrm{B}}$. The $\chi^{\prime \prime}$ data will show a peak at $f=f_{\mathrm{B}}$, which also corresponds to the inflection point in the $\chi^{\prime}$ data.

\section{EXPERIMENTAL SETUP}

To enable experiments on sensors integrated in a microfluidic channel with minimum handling of a chip, we developed a 'click-on' system providing up to 20 electrical contacts via springloaded pins as well as defining a fluidic channel over the sensors (Fig. 4) [24], [25]. The chip with dimensions of $4.7 \mathrm{~mm} \times 7.5 \mathrm{~mm}$ was placed in a well in an Al block onto which the top shown in Fig. 4a was placed and locked with two screws. The top provided the electrical contact between the contact pads on the chip and a printed circuit board on the other side containing connectors. The top also contained an inlet and an outlet connected to vertical throughholes at each end of the fluidic channel over the chip. The channel outline $(1 \mathrm{~mm} \times 5 \mathrm{~mm})$ was defined in a gasket cast in polydimethylsiloxane (PDMS). The channel height was either $1 \mathrm{~mm}$ or $0.1 \mathrm{~mm}$. The Al well in which the chip was placed was connected to a $\mathrm{Cu}$ bottom onto which a $\mathrm{Pt}$ thermometer was mounted. The base of the $\mathrm{Cu}$ bottom was placed on a Peltier element providing the heating or cooling of the system. The other side of the Peltier element interfaced with a CPU liquid cooler. The temperature of the $\mathrm{Cu}$ bottom was controlled using an LFI-3751 temperature controller (Wavelength Electronics, Inc, MT, USA). The temperature of the control thermometer was stable within $0.1^{\circ} \mathrm{C}$ and the setup covered a range of temperatures between $10^{\circ} \mathrm{C}$ and $80^{\circ} \mathrm{C}$. The temperature setpoint and ramping could be software controlled in LabView. In addition to the temperature control, either an electromagnet with an iron core ( $\pm 40 \mathrm{mT}$ field range) or a Helmholtz coil ( $\pm 11 \mathrm{mT}$ field range) could be mounted such that measurements could be performed as function of field and temperature. The mounting of a chip took less than 1 min with a success rate above $90 \%$.

The response of up to five sensors could be measured simultaneously using (typically) Stanford Research Systems SR830 lock-in amplifiers after pre-amplification using SR552 pre-amplifiers. The sensors were either biased by a constant 

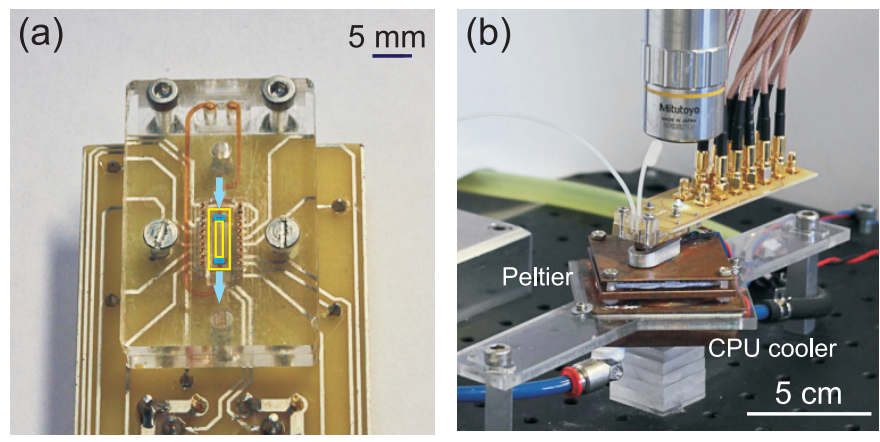

Fig. 4. (a) Picture of top integrating electrical contacts (seen along the sides of the channel), a PDMS gasket defining the outline of the fluid channel (yellow) and inlet/outlet channels (dark blue). The fluid flow is indicated by light blue arrows. (b) The top mounted on a chip in the setup with indication of the Peltier element and the CPU cooler.

current supplied by a Keithley 6221 AC and DC current source or by a constant voltage supplied by a high-fidelity audio amplifier driven by the voltage output signal from one of the lock-in amplifiers.

All measurements presented below were performed in an un-shielded laboratory environment, i.e., with neither magnetic nor electrical shielding of the sensor setup.

\section{RESULTS AND DISCUSSION}

In this section, we give an overview of the results reported in the literature on exchange-biased AMR bridge sensors. Table III presents the key characteristics of the presented sensors and lists the studies performed using the sensors. In addition to the PHEB, pPHEB and dPHEB sensor designs introduced above, the table also includes a ring-shaped version of the PHEB sensor (ring-PHEB) introduced by Oh et al. [12]. Sizelimitations on the sensor structures were discussed in [15]. Below, we first give an overview of the results obtained for magnetic field sensing and then we introduce the studies focusing on the detection of magnetic nanobeads.

\section{A. Magnetic field sensing}

Fig. 5 shows examples of field sweep measurements on a PHEB sensor of the indicated stack and geometry. The stack with $t_{\mathrm{fm}}=20 \mathrm{~nm}$ and $t_{\mathrm{Cu}}=0.6 \mathrm{~nm}$ was identified in Ref. 15 as the one with the highest low-field sensitivity of the stacks investigated $\left(t_{\mathrm{fm}}=10,20,30 \mathrm{~nm}\right.$ and $t_{\mathrm{Cu}}=0,0.3,0.6$ $\mathrm{nm})$. The field sweeps in Fig. 5 are typical for PHEB sensors showing a linear low-field region, a peak in the response at $B_{y}= \pm\left(B_{\mathrm{ex}}+B_{\mathrm{K}} / \sqrt{2}\right)$ and subsequently a signal with a decreasing magnitude. The observed low-field sensitivity, $S_{0}^{\text {obs }}$, is the slope of the $V / I_{\text {rms }}$ response at low fields.

A number of different sensor geometries and stack compositions have been investigated, see Table III. The sensor signal is proportional to the total length $\Sigma l=(m+n) l$ of a resistor in the bridge for PHEB sensors and inversely proportional to the sensor width $w$. Therefore, the highest sensitivities have been reported for sensors with high values of $\Sigma l / w$. The highest absolute value of the low-field sensitivity reported in each study is listed in Table III. However, the maximum

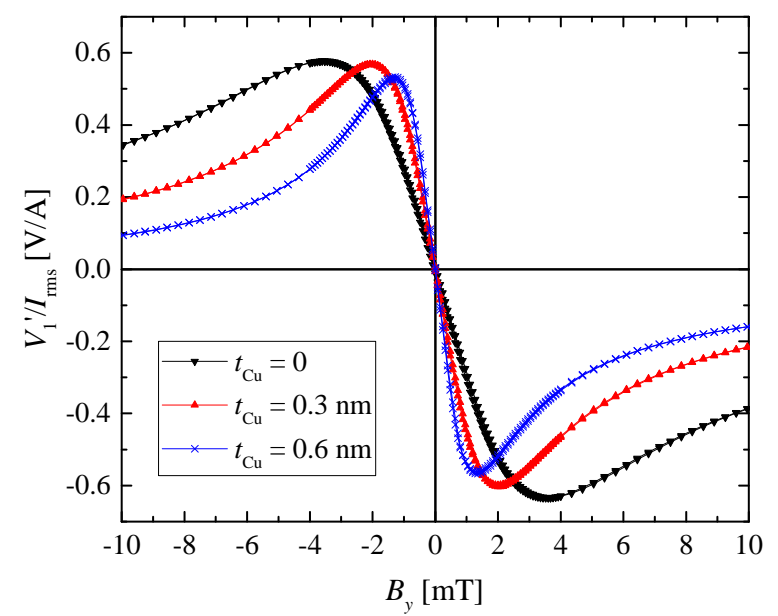

Fig. 5. Field sweeps performed on a PHEB sensor with $w=25 \mu \mathrm{m}$ and $l=250 \mu \mathrm{m}$ of the stack $\mathrm{Ta}(13 \mathrm{~nm}) / \mathrm{Ni}_{80} \mathrm{Fe}_{20}(20 \mathrm{~nm}) / \mathrm{Cu}\left(t_{\mathrm{Cu}}\right) /$ $\mathrm{Mn}_{80} \operatorname{Ir}_{20}(10 \mathrm{~nm}) / \mathrm{Ta}(3 \mathrm{~nm})$ and the indicated values of $t_{\mathrm{Cu}}$. The sensor was surrounded by magnetic stack with a gap of $3 \mu \mathrm{m}$. Measurements were performed for a current amplitude of $1 \mathrm{~mA}$. Adapted from [15] with permission of AIP publishing.

achievable signal also depends on the maximum bias current or voltage that can be applied, which may be limited due to sensor self-heating or other constraints introduced by the sensor application [14]. Furthermore, the low-field sensitivity may also be reduced by shape anisotropy of the sensor stripes [15]. Therefore, the sensor dimensions can be only be chosen within certain constraints defined by the sensor stack, the application of the sensors and the surrounding electronics.

To enable a comparison of the stack only, Table III also lists the maximum observed low-field sensitivities normalized by $\Sigma l / w$. This number provides a measure of $\Delta \rho /\left[t\left(B_{\text {ex }}+\right.\right.$ $\left.B_{\mathrm{K}}\right)$ ( (see Eq. (8) and Table II). Here, the stack with $t_{\mathrm{fm}}=$ $20 \mathrm{~nm}$ and $t_{\mathrm{Cu}}=0.6 \mathrm{~nm}$, for which the field sweep is shown in Fig. 5, had the highest value and generally the stacks in Table III including a $\mathrm{Cu}$ layer showed higher values than their counterparts without $\mathrm{Cu}$. It should be noted that the ring-PHEB sensor design theoretically does not perform as well as the PHEB sensor design with straight conductors [34] but also that the two designs are affected differently by shape anisotropy as discussed in detail in [15].

The lowest magnetic field that can be resolved by the sensors in Table III shows a complex dependence on the intrinsic noise of the sensor, on the bias current applied to the sensor, the frequencies relevant for the sensor use and on the associated detection electronics. Moreover, the practical performance of a sensor depends on the magnitude of the sensor offset and to which extent external parameters, such as temperature and external magnetic field sources, can be kept constant during experiments. Therefore, the sensors with the highest sensitivity in Table III do not necessarily have the highest field resolution. Only very few studies of the noise characteristics of exchange-biased AMR bridges and associated equipment exist [36], [37] and the noise characteristics of the sensors and associated equipment for the studies in Table III has not been investigated and is therefore not reported. It should further be noted that the biodetection sensitivity 
TABLE III

OVERVIEW OF LITERATURE ON PLANAR HALL EFFECT BRIDGE SENSORS OF THE INDICATED PRESENTED DESIGNS (PHEB, PPHEB, DPHEB) AS WELL AS A RING-SHAPED VERSION (RING-PHEB). THE 'STACK' COLUMN GIVES THE THICKNESSES OF THE NI-FE, CU (OPTIONAL) AND MN-IR LAYERS OF THE STACK AND WHEN A PARAMETER IS VARIED, THE INTERVAL OF PARAMETER VALUES IS INDICATED. THE 'DIMENSIONS' COLUMN GIVES THE LENGTH, WIDTH AND NUMBER OF STRIPES FOR THE SENSOR(S). FOR THE RING-PHEB SENSORS, THE NUMBER GIVEN IS THE LARGEST DIAMETER OF A SENSOR RING. $\rho / t$ IS THE REPORTED OR CALCULATED SHEET RESISTANCE FOR THE SENSOR STACK. $\left|S_{0}^{\text {obs }}\right|$ max IS THE LARGEST OBSERVED LOW-FIELD SENSITIVITY REPORTED FOR THE INVESTIGATED SENSORS. $\left|S_{0}^{\text {obs }}\right|_{\max } /(\Sigma l / w)$ IS THE MAXIMUM OBSERVED LOW-FIELD SENSITIVITY NORMALIZED BY THE TOTAL LENGTH/WIDTH OF ONE OF THE RESISTORS OF ONE ARM IN THE BRIDGE. IN THE 'STUDIES' COLUMN, THE KEY TOPICS OF THE STUDY ARE INDICATED. 'FIELD' INDICATES THAT FIELD SWEEPS ARE PRESENTED, 'BEADS (VOL)' INDICATES THAT MEASUREMENTS ON MAGNETIC BEAD SUSPENSIONS ARE PERFORMED, 'BEADS (SURF)' INDICATES THAT SURFACE-BASED BIOSENSING IS PERFORMED.

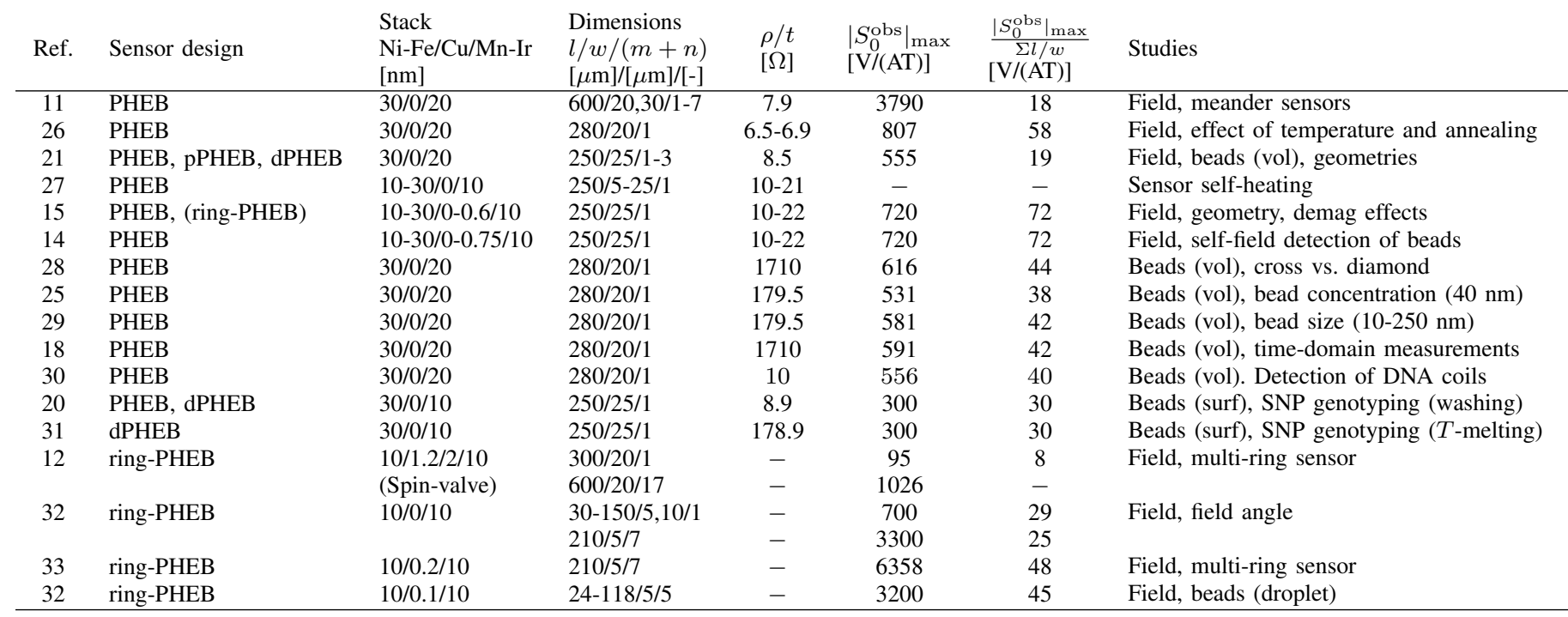

depends not only on the intrinsic sensor noise and fluctuations of external parameters but also on how the magnetic beads are placed with respect to the sensor and on the statistical fluctuations in their number and distribution [38].

\section{B. Magnetic bead sensing and biosensing}

In this section, we present a brief overview of the studies on magnetic bead detection and magnetic biosensing performed using the presented sensor designs. We will divide the discussion in volume-based measurements where the Brownian relaxation response of magnetic nanobeads is measured and surface-based measurements where the signal due to beads tethered to the sensor surface is measured. In Table III, the relevant studies are indicated as 'Beads (vol)' and 'Beads (surf)', respectively. In all studies using the PHEB, pPHEB and dPHEB sensor designs, the magnetic beads were magnetized by the self-field.

The practical performance of the PHEB, pPHEB and dPHEB designs for magnetic field detection and magnetic bead detection was investigated in [21], where they were found to exhibit the theoretically anticipated behavior summarized in Table II. The pPHEB and dPHEB designs were found to suppress the signal contribution from an external applied magnetic field by at least a factor of 100, and the dPHEB design was found to suppress the sensor self-bias offset $\left(\gamma_{0}\right.$ contribution) by at least a factor of 50 .

The effect of self-heating and the limitations on the maximum sensor bias current imposed by a maximum allowable temperature increase due to self-heating were studied in [27] and the implications for the choice of optimal sensor stack and operation conditions for self-field detection were discussed in [14].

\section{1) Volume-based measurements}

Initial work focused on demonstrating measurements of the Brownian relaxation of magnetic nanobeads. The use of bridge sensors was initiated in [28], where the additive nature of the self-field response for the PHEB design was presented and on-chip sensor measurements of the magnetic susceptibility of $40 \mathrm{~nm}$ and $50 \mathrm{~nm}$ magnetic nanobeads were compared to corresponding measurements performed in a commercial ac susceptometer. The study also demonstrated that the sensor measurements, as opposed to ac susceptibility measurements, were sensitive to the sedimentation of magnetic beads as the sensor only probes the sample volume near the sensor surface. The presented bridge design displayed a signal increase by a factor of about six compared to a corresponding cross-shaped sensor and the measurements were found to show essentially the same Brownian relaxation response as obtained in the commercial ac susceptometer.

In a subsequent study [25], the response of $40 \mathrm{~nm}$ magnetic nanobeads was studied vs. their concentration and for these beads it was found that the signal was proportional to the concentration over almost two decades and that Brownian relaxation frequencies could be extracted reliably for concentrations down to $63 \mu \mathrm{g} / \mathrm{mL}$ corresponding to a signal in $V_{2}^{\prime}$ at the Brownian relaxation frequency of $30 \mathrm{nV}$. Using Table II and the reported values of $S_{0}$ obs and $I_{\mathrm{rms}}$, we estimate that this signal corresponded to an rms magnetic bead field of 15 $\mathrm{nT}$ and note that the signal was obtained from a volume over the sensor of about $1 \mathrm{~nL}$. Furthermore, it was demonstrated 


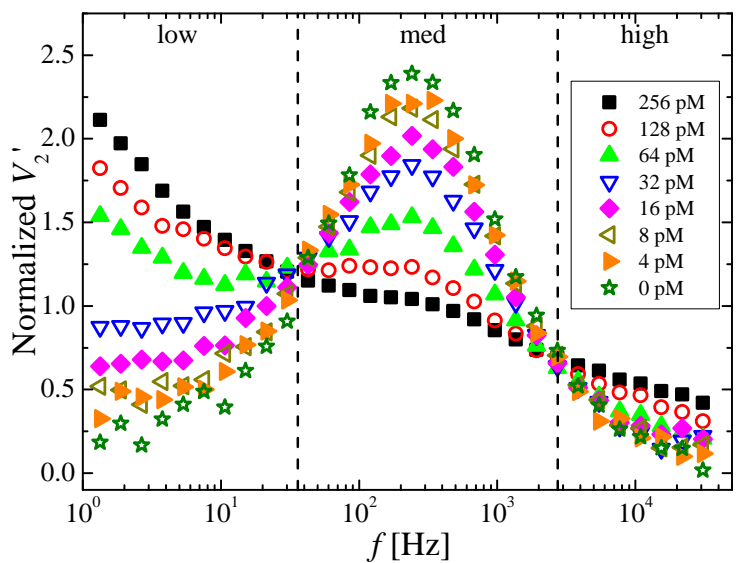

Fig. 6. Measurements of the in-phase sensor response $V_{2}^{\prime}$ for $80 \mathrm{~nm}$ functionalized magnetic nanobeads incubated with $\tilde{1} \mu \mathrm{m}$ coils of DNA formed by rolling circle amplification from a Vibrio Cholerae target at the indicated concentrations. The $V_{2}^{\prime}$-response is proportional to the $\chi^{\prime \prime}$-response. The signals from individual beads and beads bound to DNA coils are observed at medium frequencies and low frequencies, respectively. The spectra were normalized with the total signal. Reproduced from [30] with permission of John Wiley and Sons.

that on-chip measurements could be performed between $40 \mathrm{~Hz}$ and $1 \mathrm{MHz}$ in a matter of minutes.

In a further study, the ability of the sensor platform to perform reliable measurements on beads with different sizes in the range between $40 \mathrm{~nm}$ and $250 \mathrm{~nm}$ was investigated [29]. In this study, the largest beads with a size of $250 \mathrm{~nm}$ were found to provide a signal that drifted and increased with time due to sedimentation of beads on the sensor. Moreover, these beads also showed a hydrodynamic size that was significantly larger than their nominal size, possibly due to agglomeration or interaction with the sensor surface. Further, the signal-to-noise of the Brownian relaxation measurements was used to estimate theoretical limits of detection for bioanalysis and beads with a diameter of $80 \mathrm{~nm}$ were identified as optimal. In parallel, a new method for sensor measurements of the relaxation response in the time domain was presented and demonstrated for beads with sizes in the same size range [18]. This method shortened the measurement time from a few minutes to $30 \mathrm{~s}$ such that real-time measurements of the relaxation response could be performed. However, the method was also found to have limitations in terms of the bead sizes and in the window of relaxation times that could be resolved. The subsequent work was therefore still based on lock-in measurements in the frequency domain.

In a subsequent key study [30], the developed sensor technology was applied for the volume-based detection of DNA amplicons formed via a rolling circle amplification process from a bacterial DNA target from Vibrio Cholerae as well as from a Bacillus globigii bacterial spore target. The biomolecular amplification process results in long concatamers of DNA complementary to a padlock probe that recognizes the target. These coil up to form DNA coils with a diameter of about $1 \mu \mathrm{m}$. The detection of the DNA coils was based on measurements of the significant hydrodynamic size change experienced by the $80 \mathrm{~nm}$ magnetic nanobeads when they bind to the DNA coils. The binding causes these beads to contribute with a signal at a substantially lower frequency than the free beads. Figure 5 shows spectra of $V_{2}^{\prime}$ measured on-chip for the indicated concentrations of DNA coils formed from Vibrio Cholerae. Note that these spectra correspond to spectra of the out-of-phase susceptibility, $\chi^{\prime \prime}$ (see Table II) and thus display a peak at the Brownian relaxation frequency, $f_{\mathrm{B}}$ (Eq. (19)). The spectra could be divided into a regions of medium frequencies dominated by the signal from free beads and low frequencies dominated by the signal from beads bound to DNA coils. Several analysis strategies to address the lack of absolute units of the signal were investigated. The best results were obtained by taking the ratio between the total signal in the low-frequency region to that in the medium-frequency region. In the chip experiments, the sedimentation of beads bound to DNA coils was found to improve their relative signal and thus to improve the sensitivity. The lowest concentration detected of $2 \mathrm{pM}$ compared favorably to the limit of detection obtained using commercial ac susceptometers.

\section{2) Surface-based measurements}

To perform surface-based measurements of DNA interactions, selected areas on the sensor surface were functionalized with DNA detection probes. The biotinylated DNA target to be investigated was introduced in the fluid system together with $50 \mathrm{~nm}$ streptavidin magnetic beads. Hybridization of the target to the DNA detection probes enabled linking of magnetic beads to the sensor surface. After initial experimental verification of the additivity of the signals from magnetic beads bound to the surface of the different branches of the PHEB design ( $m=1, n=0$ ) [20], subsequent studies of DNA were performed using the dPHEB design with the entire bridge placed centrally in a microfluidic channel where the two top branches of the sensor bridge were functionalized with DNA detection probes and the two bottom branches were left unfunctionalized and thus functioned as a local negative reference. This design was shown to efficiently cancel the sensor offset as well as the signal from magnetic beads in suspension over the sensor such that only beads bound to the top half of the bridge via specific interactions were detected [20]. This enabled real-time measurements of the sensor response due to specific interactions under varying experimental conditions and thereby also during washing steps and temperature changes.

The magnetic beads employed in these studies showed a superparamagnetic response. The largest bead signal was found in the $V_{2}^{\prime \prime}$ signal, which is linearly related to the inphase magnetic response, $\chi^{\prime}$. This response was measured at a frequency high enough to ensure fast measurements with low noise and low enough to ensure that most of the magnetic response was in-phase with the self-field. A typical frequency used was $f=167 \mathrm{~Hz}$. In a study of the signal vs. target DNA concentration, we found that a detectable signal with a magnitude of about $15 \mathrm{nV}$ was produced for a concentration down to about $150 \mathrm{pM}$ [20]. The bead signal at this concentration corresponded to an average rms magnetic field of about $3 \mathrm{nT}$. In subsequent studies, a concentration of about $5 \mathrm{nM}$ was used, which is typical for DNA produced by 


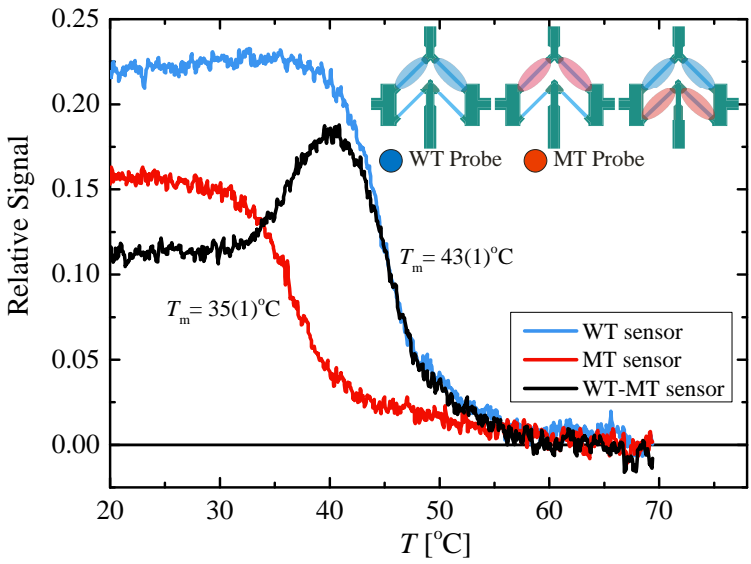

Fig. 7. Measurements of the in-phase sensor response $V_{2}^{\prime}$ for $80 \mathrm{~nm}$ functionalized magnetic nanobeads incubated with $\tilde{1} \mu \mathrm{m}$ coils of DNA formed by rolling circle amplification from a Vibrio Cholerae target at the indicated concentrations. The $V_{2}^{\prime}$-response is proportional to the $\chi^{\prime \prime}$-response. The signals from individual beads and beads bound to DNA coils are observed at medium frequencies and low frequencies, respectively. The spectra were normalized with the total signal. Adapted from [31] with permission from Elsevier.

amplification by the polymerase chain reaction (PCR).

The developed sensor platform was used for the detection and genotyping of single nucleotide polymorphisms (SNPs). In [20], we presented real-time measurements of the signal from sensors functionalized with probes targeting wild type (WT) and mutant type (MT) variants of the target during and after a stringent wash. It was demonstrated that the methods could be used for reliable genotyping of two mutation sites of the human beta globin gene. Compared to end-point detection, the real-time analysis enabled robust genotyping even for a probe that gave almost no signal at the end of the stringent washing. Thus, the approach proved to be more flexible in terms of the probe design, where probes for end-point detection often need careful design of their length to produce a detectable signal and robust genotyping.

In [31], we further demonstrated that the sensor platform could be used for SNP detection of the same targets via realtime measurements of the temperature-melting of the probetarget hybrids. In these experiments, sensors functionalized with probes matching the WT and MT target variants were incubated with the biotinylated target and streptavidin magnetic beads followed by a low-stringency washing step after which the real-time signal was measured as function of increasing temperature. Figure 7 shows the normalized and corrected realtime signal for three sensors functionalized as indicated in the inset of Fig. 7 as function of temperature after incubation with a WT target. Both the WT and MT sensors show a clear and nominally flat initial response that decays to zero upon increasing temperature. The melting temperature, $T_{\mathrm{m}}$, defined as the temperature at which the signal has decreased to half of its initial value, was found to be significantly higher for the matching MT probe than for the mismatching WT probe and the melting temperature difference thus provided a clear genotyping of the sample. The third black curve in the figure shows the response for a sensor bridge functionalized with WT probes on its top half and MT probes on its bottom half. A clear peak in the corresponding WT-MT signal was observed and the signal matched that obtained by subtraction of the signals from the WT and MT sensors. This demonstrated that the genotyping for this mutation could be obtained using only one sensor bridge.

In addition to the DNA detection studies mentioned above, ring-PHEB sensors have been applied to detect $1-2 \mu \mathrm{L}$ of magnetic bead suspensions deposited on the sensor surface [35]. These measurements were carried out using lock-in detection of the sensor response to an external magnetizing field with a $1 \mathrm{mT}$ ac component and a $-3 \mathrm{mT}$ dc component applied at an angle of $20^{\circ}$ to the direction of the exchange-pinning field, where the dc field served to increase the sensitivity. The lowest amount of beads detected was estimated to correspond to a magnetic moment of $4 \times 10^{-16} \mathrm{Am}^{2}$. Using their reported sensitivity and signal change, we calculate that the bead signal in this case corresponded to an average magnetic field of about $6 \mathrm{nT}$.

\section{Outlook}

The presented sensors have demonstrated detection of magnetic fields down to the nT range. For example in Section IV-B2 a $15 \mathrm{nV}$ signal due to surface-bound magnetic beads was resolved [31]. Considering that the thermal voltage noise of this sensor with a resistance of $89 \Omega$ is $V_{n} / \sqrt{\Delta f}=$ $\sqrt{4 k_{\mathrm{B}} T R} \approx 1.2 \mathrm{nV} / \mathrm{Hz}^{1 / 2}$ and that experiments were performed at $f=167 \mathrm{~Hz}$ with $\Delta f \approx 1 \mathrm{~Hz}$, it is likely that the main limiting factor in the presented studies was noise in the electronics used for the readout and/or fluctuations of external parameters such as temperature and the background field in the unshielded laboratory environment where the measurements were performed. Thus, the presented results can likely be further improved by use of more optimal operation conditions. For such an optimization, investigations of the sensor noise characteristics as function of bias current are needed to find the optimum combination of sensor stripe length and bias current to optimize the balance between the sensor signal, the $1 / f$ noise and the thermal noise. At present only few studies of exchange-biased AMR bridges exist and there is a general need for more studies of sensors with different stacks and geometries to establish reliable values of, e.g., the Hooge parameter that characterizes the $1 / f$ noise [36], [37].

As opposed to barberpole AMR sensors, which can be designed to dynamically self-correct for an offset in the sensor response such that absolute field measurements can be performed [4], the presented sensors suffer from a temperaturedependent offset due to a slight unbalance in the bridge. At present, it is yet to be explored to which extent this offset can be nullified and compensated for. Therefore, the present sensors are best used for measurements of field changes, where an offset is a smaller problem.

The sensors have proven their ability to detect small amounts of magnetic beads both in volume-based and surfacebased detection formats and that the sensor can be integrated in a flexible lab-on-a-chip platform. The presented detection scheme using the sensor self-field allows for detection with 
no signal cancellation effects due to the position of the beads with respect to the sensor. The presented scheme allows for comparatively fast measurements in a compact format, which is capable of covering a wide range of frequencies (dc to $\mathrm{MHz}$ ). The sensor system is flexible and can be integrated in lab-on-a-chip systems or other systems for dynamic magnetic measurements. Drawbacks of the system are the limited potential to make low-cost disposable systems and that only relative magnetic susceptibility measurements can be performed. However, as demonstrated, the sensor design space is large and highly flexible offering opportunities to tailor the design and operation of sensors to specific application and to include built-in reference structures.

\section{CONCLUSIONS}

We have reviewed the theory of operation of exchangebiased AMR bridge sensors, also termed planar Hall effect bridge sensors, starting from simple sensor construction elements to how these can be combined to form sensors tailored towards magnetic field sensing, sensing of magnetic beads and differential sensing. We have focused on the special application where the sensors are used to detect magnetic beads being magnetized by the sensor self-field arising from the bias current passed through the sensor and we have presented theoretical expressions for the signals that can be measured using lock-in detection. We have introduced the setup integrating electrical contacts and a fluid channel in a simple 'click-on' system. We have reviewed the literature on magnetic field sensing using planar Hall effect bridge sensors and a simple overview of the characteristics and performance of the presented designs was given. We further reviewed the literature on applications of the sensors for magnetic bead measurements and biosensing either in volume-based or surface-based formats and gave an outlook on challenges and opportunities for these sensors.

\section{ACKNOWLEDGMENT}

Support from the Danish Council for Independent Research (G.R. Postdoc project, DFF-4005-00116) and EU FP7 grant No. 604448-NanoMag is gratefully acknowledged.

\section{REFERENCES}

[1] K. J. M. Eijkel, and J. H. J. Fluitman, "Optimization of the Response of Magnetoresistive Elements," IEEE Trans. Magn., vol. 26, p. 311-321, 1990.

[2] J. E. Lenz, G. F. Rouse, L. K. Strandjord, B. B. Pant, A. Metze, H. B. French, E. T. Benser, and D. R. Krahn,"A high-sensitivity magnetoresistive sensor," Proc. 4th IEEE Solid-State Sens. Actuat. Workshop Tech. Digest, p. 114-117, 1998.

[3] F. Montaigne, A. Schuhl, F. N. van Dau, and A. Encinas, ,"Development of magnetoresistive sensors based on planar Hall effect for applications to microcompass," Sensors and Actuators, vol. 81, p. 324-327, 2000.

[4] H. Hauser and M. Tondra, "Magnetoresistors," in Magnetic sensors and magnetometers, P. Ripka, Ed. Norwood, MA, USA: Artech House, 2001, ch. 4, pp. 129-172.

[5] P. P. Freitas, H. Ferreira, D. L. Graham, L. A. Clarke, M. D. Amaral, V. Martins, L. Fonseca, and J. S. Cabral, "Magnetoresistive DNA chips," in Magnetoelectronics, 1st ed., M. Johnson, Ed. Kidlington, Oxford, UK: Elsevier, 2004, ch. 7, pp. 331-373.

[6] A. Grosz, V. Mor, E. Papermo, S. Amrusi, I. Faivinov, M. Schultz, and L. Klein,"Planar Hall Effect Sensors with Subnanotesla Resolution," IEEE Magn. Lett., vol. 4, 6500104, 2013.
[7] A. Grosz, V. Mor, S. Amrusi, I. Faivinov, E. Paperno, and L. Klein, “A High-Resolution Planar Hall Effect Magnetometer for Ultra-Low Frequencies," IEEE Sensors J., vol. 16, 3224-3230, 2016.

[8] L. Jogschies, D. Klaas, R. Kruppe, J. Rittinger, P. Taptimthong, A. Wienecke, L. Rissing, and M. C. Wurz, "Recent Developments of Magnetoresistive Sensors for Industrial Applications," Sensors, vol. 15, pp. $28665-28689,2015$.

[9] L. Ejsing, M. F. Hansen, A. K. Menon, H. A. Ferreira, D. L. Graham, and P. P. Freitas, "Planar Hall effect sensor for magnetic micro- and nanobead detection," Appl. Phys. Lett., vol. 84, p. 4729, 2004.

[10] T. Q. Hung, S. Oh, S. Anandakumar, J. R. Jeong, D. Y. Kim, and C. Kim, "Optimization of the multilayer structures for a high field-sensitivity biochip sensor based on the planar Hall effect," IEEE Trans. Magn., vol. 45, pp. 4518-4521, 2009.

[11] A. D. Henriksen, B. T. Dalslet, D. H. Skieller, K. H. Lee, F. Okkels, and M. F. Hansen, "Planar Hall effect bridge magnetic field sensors," Appl. Phys. Lett., vol. 97, p. 013507, 2010.

[12] S. Oh, P. B. Patil, T. Q. Hung, B. Lim, M. Takahashi, D. Y. Kim, and C. Kim, "Hybrid AMR/PHR ring sensor," Solid State Commun., vol. 151, pp. 1248-1251, 2011.

[13] T. Q. Hung, S. Oh, B. Sinha, J.-R. Jeong, D.-Y. Kim, and C. Kim, "High field-sensitivity planar Hall sensor based on $\mathrm{NiFe} / \mathrm{Cu} / \mathrm{IrMn}$ trilayer structure," J. Appl. Phys., vol. 107, p. 09E715, 2010.

[14] A. D. Henriksen, G. Rizzi, and M. F. Hansen, "Planar Hall effect bridge sensors with $\mathrm{NiFe} / \mathrm{Cu} / \mathrm{IrMn}$ stack optimized for self-field magnetic bead detection," J. Appl. Phys., vol. 119, p. 093910, 2016.

[15] A. D. Henriksen, G. Rizzi, and M. F. Hansen,"Experimental comparison of ring and diamond shaped planar Hall effect bridge magnetic field sensors," J. Appl. Phys., vol. 118, p. 103901, 2015.

[16] A. D. Henriksen, S. X. Wang, and M. F. Hansen, "On the importance of sensor height variation for detection of magnetic labels by magnetoresistive sensors," Sci. Rep., vol. 5, p. 12282, 2016.

[17] C. D. Damsgaard and M. F. Hansen, "Theoretical study of in-plane response of magnetic field sensor to magnetic beads in an in-plane homogeneous field," J. Appl. Phys., vol. 103, p. 064512, 2008.

[18] F. W. Østerberg, G. Rizzi, and M. F. Hansen, "On-chip Brownian relaxation measurements of magnetic nanobeads in the time domain," $J$. Appl. Phys., vol. 113, p. 234508, 2013.

[19] T. B. G. Hansen, C. D. Damsgaard, B. T. Dalslet, and M. F. Hansen, "Theoretical study of in-plane response of magnetic field sensor to magnetic beads magnetized by the sensor self-field," J. Appl. Phys., vol. 107 , p. 124511, 2010.

[20] G. Rizzi, F. W. Østerberg, M. Dufva, and M. F. Hansen, "Magnetoresistive sensor for real-time single nucleotide polymorphism genotyping," Biosens. Bioelectron., vol. 52, pp. 445-451, 2014.

[21] F. W. Østerberg, G. Rizzi, A. D. Henriksen, and M. F. Hansen, "Planar Hall effect bridge geometries optimized for magnetic bead detection," J. Appl. Phys., vol. 115, p. 184505, 2014.

[22] P. Debye, Polar molecules. Chemical Catalogue Co, 1929

[23] K. S. Cole and R. H. Cole, "Dispersion and Absorption in Dielectrics I. Alternating Current Characteristics," J. Chem. Phys., vol. 9, p. 341, 1941.

[24] F. W. Østerberg, B. Dalslet, D. Snakenborg, C. Johansson, and M. F. Hansen, "Chip-Based Measurements of Brownian Relaxation of Magnetic Beads Using a Planar Hall Effect Magnetic Field Sensor," AIP Conf. Proc., vol. 1311, pp. 176-183, 2010.

[25] F. W. Østerberg, G. Rizzi, and M. F. Hansen, "On-chip measurements of Brownian relaxation vs. concentration of $40 \mathrm{~nm}$ magnetic beads," $J$. Appl. Phys., vol. 112, p. 124512, 2013.

[26] G. Rizzi, N. C. Lundtoft, F. W. Østerberg, and M. F. Hansen, "Reversible and Irreversible Temperature-induced Changes in Exchange-biased Planar Hall Effect Bridge (PHEB) Magnetic Field Sensors," Sensors \& Transducers, vol. 15, pp. 22-34, 2012.

[27] A. D. Henriksen, G. Rizzi, F. W. Østerberg, and M. F. Hansen, "Optimization of magnetoresistive sensor current for on-chip magnetic bead detection using the sensor self-field," J. Magn. Magn. Mater, vol. 280, pp. 209-214, 2015.

[28] F. W. Østerberg, G. Rizzi, T. Zardán Gómez de la Torre, M. Strömberg, M. Strømme, P. Svedlindh, and M. F. Hansen, "Measurements of Brownian relaxation of magnetic nanobeads using planar Hall effect bridge sensors," Biosens. Bioelectron., vol. 40, no. 1, pp. 147-152, 2013

[29] F. W. Østerberg, G. Rizzi, and M. F. Hansen, "On-chip measurements of Brownian relaxation of magnetic beads with diameters from $10 \mathrm{~nm}$ to $250 \mathrm{~nm}$, , J. Appl. Phys., vol. 113, p. 154507, 2013.

[30] F. W. Østerberg, G. Rizzi, M. Donolato, R. S. Bejhed, A. Mezger, M. Strömberg, M. Nilsson, M. Strømme, P. Svedlindh, and M. F. Hansen, "On-Chip Detection of Rolling Circle Amplified DNA Molecules from
758

759 760 
Bacillus Globigii Spores and Vibrio Cholerae,’ Small, vol. 10, pp. 2877 2882, 2014.

[31] G. Rizzi, F. W. Østerberg, A. D. Henriksen, M. Dufva, and M. F. Hansen "On-chip magnetic bead-based DNA melting curve analysis using a magnetoresistive sensor," J. Magn. Magn. Mater, vol. 380, pp. 215220, 2015.

[32] B. Sinha, S. Oh, T. Sri Ramulu, J. Lim, D. Y. Kim, and C. G. Kim "Planar Hall Effect Ring Sensors for High Field-Sensitivity," Adv. Mat. Res., vol. 317, pp. 1136-1140, 2011.

[33] B. Sinha, T. Quang Hung, T. Sri Ramulu, S. Oh, K. Kim, D.-Y. Kim, F. Terki, and C. Kim, "Planar Hall resistance ring sensor based on NiFe/Cu/IrMn trilayer structure," J. Appl. Phys., vol. 113, p. 063903, 2013.

[34] F. W. Østerberg, A. D. Henriksen, G. Rizzi, and M. F. Hansen, "Comment on 'Planar Hall resistance ring sensor based on $\mathrm{NiFe} / \mathrm{Cu} / \mathrm{IrMn}$ trilayer structure' [J. Appl. Phys. 113, 063903 (2013)]," J. Appl. Phys., vol. 114, p. 106101, 2013.

[35] T. Q. Hung, F. Terki, S. Kamara, K. Kim, S. Charar, and C. Kim, "Planar Hall ring sensor for ultra-low magnetic moment sensing," J. Appl. Phys., vol. 117, p. 154505, 2015.

[36] A. Persson, R. S. Bejhed, H. Nguyen, K. Gunnarsson, B. T. Dalslet, F. W. Østerberg, M. F. Hansen, P. Svedlindh, "Low-frequency noise in planar Hall effect bridge sensors," Sensor Actuat. A - Phys, vol. 171, p. 212-218, 2011.

[37] A. Persson, R. S. Bejhed, F. W. Østerberg, K. Gunnarsson, H. Nguyen, G. Rizzi, M. F. Hansen, P. Svedlindh, "Modelling and design of planar Hall effect bridge sensors for low-frequency applications," Sensor Actuat. A - Phys, vol. 189, p. 459-465, 2013.

[38] A. D. Henriksen, M. W. H. Ley, H. Flyvbjerg, and M. F. Hansen, "Configurational statistics of magnetic bead detection with magnetoresistive sensors", PLoS ONE, vol. 10, e0141115, 2015.
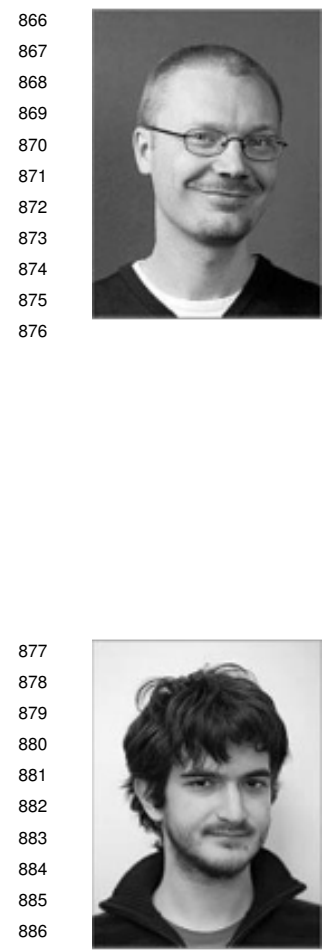

Mikkel Fougt Hansen, born in 1971, obtained his M.Sc. degree in physics from University of Copenhagen in 1995 and his Ph.D. degree in physics in 1998 from the Technical University of Denmark (DTU). This was followed by postdoctoral positions at Uppsala University and at University of California, San Diego. Since 2002 he has been associate professor at DTU Nanotech at DTU. His present research interests include lab-on-a-chip systems, magnetic nanoparticles, magnetic biosensors and applications thereof.

Giovanni Rizzi, born in 1986 in Milano, Italy, received his M.Sc. degree in Engineering Physics in 2010 at Politecnico di Milano and his Ph.D. degree in physics in 2014 from DTU Nanotech at the Technical University of Denmark, where he is currently also working in a postdoctoral position. His research is focused on developing magnetoresistive sensors as a platform for DNA analysis. His present research interests include lab-on-a-chip systems, biosensors and magnetic sensors. 